\title{
Multi-Node Cooperative Resource Allocation to Improve Coverage Area in Wireless Networks
}

\author{
Ahmed K. Sadek, Zhu Han, and K. J. Ray Liu \\ Department of Electrical and Computer Engineering, and Institute for Systems Research \\ University of Maryland, College Park, MD 20742, USA. \{aksadek, hanzhu, kjrliu\}@eng.umd.edu
}

\begin{abstract}
It is of great importance for service providers to improve the coverage area without cost of more infrastructure in wireless networks especially in rural areas. Recently, cooperative communication has brought a new communication paradigm. In this paper, multi-node cooperative resource allocation is studied to schedule the transmission time slots so as to maximize the network overall rate (or the coverage area) under the Quality of Services constraints. To optimize the performance, first, the performance of the multi-node cooperative transmission is analyzed. Then a protocol is constructed between the mobiles and the base station for the resource allocation. From the simulation results, the proposed scheme can increase the user's rate by $5 \%$ for large cell sizes and improve the coverage by $180 \%$, compared with the traditional scheme without cooperative transmission.
\end{abstract}

\section{INTRODUCTION}

Over the past few decades, wireless communications and networking have witnessed an unprecedented growth. The growing demands require the service providers to provide reliable wireless communication anytime anywhere. This poses challenges for deployment of wireless networks, especially in rural area. Since the power of the mobile is limited, it requires enough number of the base stations to maintain the coverage. However, the base stations are expensive to construct. Moreover, the density of the users are also small for rural area and it is hard to obtain the profits to cover the cost of deploying the base stations. Consequently, there is a tradeoff for improving service coverage and reducing the cost of constructing base stations. So expanding the service coverage in rural wireless networks without increasing the cost of infrastructure has drawn great attention from wireless service providers and academia.

Resource allocation is a general strategy to improve this tradeoff by optimally utilizing the limited radio resources such as bandwidth. Many research works have been proposed for efficient resource allocation. Power control continuously adjusts the transmitting power so as to maintain the received link quality [1]. Adaptive modulation, adaptive coding, and power control can be combined together to optimize the resource usages [2]. Other adaptation such as turbo code and space time coding are discussed for different systems such as TDMA, CDMA, and OFDM in [3]. Some fairness issues are analyzed in [4].

Cooperative communication has brought a new communication paradigm. The basic idea is to explore the broadcast nature of wireless networks. The relay nodes can hear the direct transmission from the source to the destination, and then

\footnotetext{
${ }^{1}$ This work was supported in part by U.S. Army Research Laboratory under Cooperative Agreement DAAD 190120011.
}

transmit the information for the source on other channels. By exploring cooperative communication, the multipath/multiuser diversity is employed. Moreover the source and relay nodes form a virtual transmit antenna array, so many multiple input multiple output (MIMO) results can be applied. In the literature, the cooperative transmission scheme and implementation algorithms were proposed in [5], [6], [7], [8], where transmission protocols of cooperative communications can be classified into different approaches and performances are analyzed in terms of outage probabilities. Recent work in [10] presented theoretical characterization and analysis for a class of multinode cooperative protocols. This work also provided optimal power allocation for the multi-node relay problem based on an approximate expression for the SER.

In this paper, we try to improve the coverage problem in rural wireless networks by employing cooperative communication. The basic idea is to find the relay route to the base station so as to optimize the performance. The problem is formulated to maximize the system overall rate under the constraint of a maximum bit error rate (BER), maximal number of relays in the relay route, and power constraint. The problem is solved by a protocol between the users and the base station. From the simulation results, the proposed scheme can increase the user's rate by $5 \%$ for large cell sizes and improve the coverage by $180 \%$, compared with the traditional scheme without cooperation transmission.

This paper is organized as follows: In Section II, we have the system model for the multi-node cooperative communication. In Section III, we provide SER performance analysis for the system. The proposed cooperative resource allocation system is described in Section IV. Simulation results are conducted in Section V and conclusions are drawn in Section VI.

\section{Multi-node Cooperative System Model}

We consider a cellular system in which the users can cooperate in the uplink channel to forward each others data. The cooperation strategy employs a decode-and-forward protocol at the relaying nodes. In each phase of the cooperation protocol, if the node decodes correctly, it retransmits the information to the basestation, otherwise it remains idle. This cooperation strategy can be viewed as a repetition coding. The wireless link between any two nodes in the network is modeled as a Rayleigh fading narrowband channel with additive white Gaussian noise (AWGN) with variance $\mathcal{N}_{o}$. The channel fades for different links are assumed to be statistically independent. For medium access, the relays are assumed to transmit over orthogonal channels. 
The cooperation strategy we are considering employs a decode-and-forward protocol at the relaying nodes. In each phase of the cooperation protocol, if the node decodes correctly, it retransmits the information to the destination, otherwise it remains idle. We assume that the relays can judge whether it can correctly decode the information or not, by applying a SNR threshold over the received symbols for example. ${ }^{1}$ This is different from the cooperation scheme proposed in [9], which assumes that a decoding error at any intermediate terminal results in an error at the destination. Various scenarios for the cooperation among the relays can be implemented. A general cooperation scenario, denoted as $\mathcal{C}(m) \quad(1 \leq m \leq N-1)$, where $N$ is the number of relays assigned to help the source, can be implemented in which each relay combines the signals received from the $m$ previous relays along with that received from the source. Due to the symmetry of the problem among users, we only model user's $k$ signalling (which we denote by the source) and we denote a user that is assigned to help another user forward his data by a relay. The terms basestation and destination are used to denote the ultimate receiver.

For a general scheme $\mathcal{C}(m)$, the cooperation protocol has $(N+1)$ Phases. In Phase 1 , the source transmits the information, and the received signal at the destination and the $i$-th relay can be modeled respectively as

$$
\begin{aligned}
& y_{s, d}=\sqrt{P_{0}} h_{s, d} x+n_{s, d}, \\
& y_{s, r_{i}}=\sqrt{P_{0}} h_{s, r_{i}} x+n_{s, r_{i}}, \quad 1 \leq i \leq N,
\end{aligned}
$$

where $P_{0}$ is the power transmitted at the source, $x$ is the transmitted symbol with unit power, $h_{s, d} \sim C N\left(0, \sigma_{s, d}^{2}\right)$ and $h_{s, r_{i}} \sim C N\left(0, \sigma_{s, r_{i}}^{2}\right)$ are the channel fading coefficients between the source and the destination, and $i$-th relay, respectively, and $C N\left(\alpha, \sigma^{2}\right)$ denotes a circularly symmetric complex Gaussian random variable with mean $\alpha$ and variance $\sigma^{2}$. The terms $n_{s, d}$ and $n_{s, r_{i}}$ denote the AWGN. In Phase 2, if the first relay correctly decodes, it forwards the decoded symbol with power $P_{1}$ to the destination, otherwise it remains idle. Generally in Phase $l, 2 \leq l \leq N$, the $l$-th relay combines the received signals from the source and the previous $\min \{m, l-1\}$ relays using a maximal-ratio-combiner (MRC) as

$$
y_{r_{l}}=\sqrt{P_{0}} h_{s, r_{l}}^{*} y_{s, r_{l}}+\sum_{i=\max (1, l-m)}^{l-1} \sqrt{\hat{P}_{i}} h_{r_{i}, r_{l}}^{*} y_{r_{i}, r_{l}},
$$

where $h_{r_{i}, r_{l}} \sim C N\left(0, \sigma_{r_{i}, r_{l}}^{2}\right)$ is the channel gain between the $i$-th and the $l$-th relays. In (3), $y_{r_{i}, r_{l}}$ denotes the signal received at the $l$-th relay from the $i$-th relay, and can be modeled as

$$
y_{r_{i}, r_{l}}=\sqrt{\hat{P}_{i}} h_{r_{i}, r_{l}} x+n_{r_{i}, r_{l}}
$$

where $\hat{P}_{i}$ is the power transmitted at relay $i$ in Phase $(i+$ 1 ), and $\hat{P}_{i}=P_{i}$ if relay $i$ correctly decodes the transmitted symbol, otherwise $\hat{P}_{i}=0$. The $l$-th relay uses $y_{r_{l}}$ in (3) as the detection statistics. If relay $l$ decodes correctly it transmits

\footnotetext{
${ }^{1}$ Although there will always be an event of error propagation, we assume that by adjusting the SNR threshold the event of error propagation will be negligible
}

with power $\hat{P}_{l}=P_{l}$ in Phase $(l+1)$, otherwise it remains idle. Finally, in Phase $(N+1)$, the destination coherently combines all of the received signals using an MRC as follows

$$
y_{d}=\sqrt{P_{0}} h_{s, d}^{*} y_{s, d}+\sum_{i=1}^{N} \sqrt{\hat{P}_{i}} h_{r_{i}, d}^{*} y_{r_{i}, d} .
$$

In all the cooperation scenarios considered, the total transmitted power is fixed as $P_{0}+\sum_{i=1}^{N} P_{i}=P$.

\section{EXACT SER PERFORMANCE ANALYSIS}

In this section, we present SER performance analysis for a general cooperative scheme $\mathcal{C}(m)$ for any $1 \leq m \leq N-$ 1. First, we introduce some terminologies that will be used throughout the paper. For a given transmission, each relay can be in one of two states: either it decoded correctly or not. Let us define a $1 \times n, 1 \leq n \leq N$, vector $\mathbf{S}_{n}$ to represent the states of the first $n$ relays for a given transmission. The $k$-th entry of the vector $\mathbf{S}_{n}$ denotes the state of the $k$-th relay, $1 \leq k \leq n$, as follows

$$
S_{n}[k]= \begin{cases}1 & \text { if relay } k \text { correctly decodes } \\ 0 & \text { otherwise }\end{cases}
$$

Since the decimal value of the binary vector $\mathbf{S}_{n}$ can take on values from 0 to $2^{n}-1$, for convenience we denote the state of the network by an integer decimal number. Let $\mathbf{B}_{x, n}$ be the $1 \times$ $n$ binary representation of a decimal number $x$, with $B_{x, n}[1]$ being the most significant bit. So, $\mathbf{S}_{N}=\mathbf{B}_{x, N}$ indicates that the $k$-th relay, $1 \leq k \leq N$, is in state $S_{N}[k]=B_{x, N}[k]$.

We consider a general cooperation scheme $\mathcal{C}(m), 1 \leq m \leq$ $N-1$, in which the $k$-th $(1 \leq k \leq N)$ relay coherently combines the signals received from the source along with the signals received from the previous $\min \{m, k-1\}$ relays. The state of each relay in this scheme depends on the states of the previous $m$ relays, i.e., whether these relays decoded correctly or not. This is due to the fact that the number of signals received at each relay depends on the number of relays that decoded correctly from the previous $m$ relays. Hence, the joint probability of the states is given by

$$
\begin{aligned}
P\left(\mathbf{S}_{N}\right)= & P\left(S_{N}[1]\right) \cdot P\left(S_{N}[2] \mid S_{N}[1]\right) \cdots \\
& \cdot P\left(S_{N}[N] \mid S_{N}[N-1], \cdots, S_{N}[N-m]\right) .
\end{aligned}
$$

Conditioning on the network state, which can take on $2^{N}$ values, the probability of error at the destination given the channel state information (CSI) can be calculated using the law of total probability as follows

$$
P_{e \mid C S I}=\sum_{i=0}^{2^{N}-1} \operatorname{Pr}\left(e \mid \mathbf{S}_{N}=\mathbf{B}_{i, N}\right) \operatorname{Pr}\left(\mathbf{S}_{N}=\mathbf{B}_{i, N}\right),
$$

where $e$ denotes the event that the destination decoded in error, and the above summation is over all possible network states.

Now, let us compute the terms in (8). The destination collects the copies of the signal transmitted in the previous phases using a MRC (5). The resulting SNR at the destination can be computed as

$$
S N R_{d}=\frac{P_{0}\left|h_{s, d}\right|^{2}+\sum_{j=1}^{N} P_{j} B_{i, N}[j]\left|h_{r_{j}, d}\right|^{2}}{\mathcal{N}_{o}},
$$


where $B_{i, N}[j]$ takes value 1 or 0 and determines whether the $j$ th relay has decoded correctly or not. The $k$-th relay coherently combines the signals received from the source and the previous $m$ relays. The resulting SNR can be calculated as

$S N R_{r_{k}}^{m}=\frac{P_{0}\left|h_{s, r_{k}}\right|^{2}+\sum_{j=\max (1, k-m)}^{k-1} P_{j} B_{i, N}[j]\left|h_{r_{j}, r_{k}}\right|^{2}}{\mathcal{N}_{o}}$.

If M-PSK modulation is used in the system, with instantaneous SNR $\gamma$, the SER given the channel state information is given by [12]

$$
P_{C S I}^{P S K}=\Psi_{P S K}(\gamma) \triangleq \frac{1}{\pi} \int_{0}^{(M-1) \pi / M} \exp \left(-\frac{b_{P S K} \gamma}{\sin ^{2}(\theta)}\right) d \theta,
$$

where $b_{P S K}=\sin ^{2}(\pi / M)$. If M-QAM ( $M=2^{k}$ with $k$ even) modulation is used in the system, the corresponding conditional SER can be expressed as [12]

$P_{C S I}^{Q A M}=\Psi_{Q A M}(\gamma) \triangleq 4 C Q\left(\sqrt{b_{Q A M} \gamma}\right)-4 C^{2} Q^{2}\left(\sqrt{b_{Q A M} \gamma}\right)$,

in which $C=1-\frac{1}{\sqrt{M}}, b_{Q A M}=3 /(M-1)$, and $Q(x)$ is the complementary distribution function $(\mathrm{CDF})$ of the Gaussian distribution, and is defined as $Q(x)=\frac{1}{\sqrt{2 \pi}} \int_{x}^{\infty} \exp \left(-\frac{t^{2}}{2}\right) d t$.

Let us focus on computing the SER in the case of M-PSK modulation, and the same procedure is applicable for the case of M-QAM modulation. From (9), and for a given network state $\mathbf{S}_{N}=\mathbf{B}_{i, N}$, the conditional SER at the destination can be computed as

$$
\operatorname{Pr}\left(e \mid \mathbf{S}_{N}=\mathbf{B}_{i, N}\right)=\Psi_{P S K}\left(S N R_{d}\right) .
$$

Denote the conditional probability that the $k$-th relay is in state $B_{i, N}[k]$ given the states of the previous $m$ relays by $P_{k, i}^{m}$. From (10), this probability can be computed as follows

$$
P_{k, i}^{m}= \begin{cases}\Psi_{P S K}\left(S N R_{r_{k}}^{m}\right), & \text { if } B_{i, N}[k]=0 \\ 1-\Psi_{P S K}\left(S N R_{r_{k}}^{m}\right), & \text { if } B_{i, N}[k]=1\end{cases}
$$

To compute the average SER, we need to average the probability in (8) over all channel realizations, i.e., $P_{S E R}(m)=$ $E_{C S I}\left[P_{e \mid C S I}\right]$. Using (7), (13), and (14), $P_{S E R}(m)$ can be expanded as follows

$$
P_{S E R}(m)=\sum_{i=0}^{2^{N}-1} E_{C S I}\left[\Psi_{P S K}\left(S N R_{d}\right) \prod_{k=1}^{N} P_{k, i}^{m}\right] .
$$

Since the channel fades between different pairs of nodes in the network are statistically independent by the virtue that different nodes are not co-located, the quantities inside the expectation operator in the above equation are functions of independent random variables, and thus can be further decomposed as

$P_{S E R}(m)=\sum_{i=0}^{2^{N}-1}\left\{E_{C S I}\left[\Psi_{P S K}\left(S N R_{d}\right)\right] \prod_{k=1}^{N} E_{C S I}\left[P_{k, i}^{m}\right]\right\}$

The above analysis is applicable to the M-QAM case by changing the function $\Psi_{P S K}(\cdot)$ into $\Psi_{Q A M}(\cdot)$. The exact SER can be determined in the following theorem and the proof is omitted for lack of space.

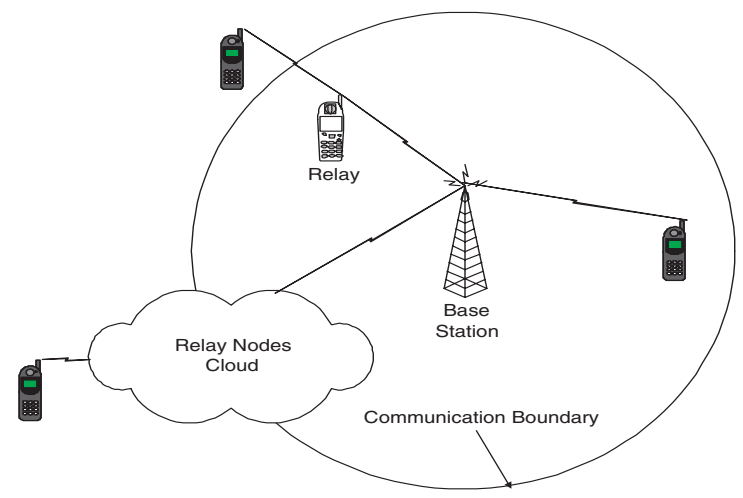

Fig. 1: System Illustration

Theorem 1: The SER of an $N$-relay decode-and-forward cooperative diversity network utilizing protocol $\mathcal{C}(m), 1 \leq$ $m \leq N-1$, and M-PSK or M-QAM modulation is given by

$$
\begin{aligned}
P_{S E R}(m)= & \sum_{i=0}^{2^{N}-1} F_{q}\left[\left(1+\frac{b_{q} P_{0} \sigma_{s, d}^{2}}{\mathcal{N}_{o} \sin ^{2}(\theta)}\right)\right. \\
& \left.\prod_{j=1}^{N}\left(1+\frac{b_{q} B_{i, N}[j] P_{j} \sigma_{r_{j}, d}^{2}}{\mathcal{N}_{o} \sin ^{2}(\theta)}\right)\right] \prod_{k=1}^{N} G_{k}^{m}\left(B_{i, N}[k]\right),
\end{aligned}
$$

where $q=1$ and $q=2$ correspond to M-PSK and M-QAM, respectively.

The constants $b_{1}=b_{p s k}, b_{2}=\frac{b_{Q A M}}{2}$, and the function $F_{q}(\cdot)$ is defined as

$$
\begin{aligned}
& F_{1}(x(\theta))=\frac{1}{\pi} \int_{0}^{(M-1) \pi / M} \frac{1}{x(\theta)} d \theta \\
& F_{2}(x(\theta))=\frac{4 C}{\pi} \int_{0}^{\pi / 2} \frac{1}{x(\theta)} d \theta-\frac{4 C^{2}}{\pi} \int_{0}^{\pi / 4} \frac{1}{x(\theta)} d \theta .
\end{aligned}
$$

In $(17), G_{k}^{m}(1)=1-G_{k}^{m}(0) \quad$ and $G_{k}^{m}(0) \quad=\quad F_{q}\left[\left(1+\frac{b_{q} P_{0} \sigma_{s, r_{k}}^{2}}{\mathcal{N}_{o} \sin ^{2}(\theta)}\right) \times\right.$ $\left.\prod_{j=\max (1, k-m)}^{k-1}\left(1+\frac{b_{q} B_{i, N}[j] P_{j} \sigma_{r_{j}, r_{k}}^{2}}{\mathcal{N}_{o} \sin ^{2}(\theta)}\right)\right]$.

It has been shown in [10] that the class of protocols $\{\mathcal{C}(m)\}$ share the same asymptotic SER performance at enough high $\mathrm{SNR}$. Hence, in the sequel we only focus on protocol $\mathcal{C}(1)$.

\section{Cooperative Resource Allocation System}

In this section, we explain the multi-node cooperative resource allocation system and develop the corresponding communication protocols. Fig. 1 shows the illustration of the proposed system. Since the mobile is powered by battery, the maximal transmit power is limited. So there exists a communication boundary where the basic link quality can not be maintained by the direct transmission. By exploring the cooperation communication, if the user is far away from the base station, some mobiles serving as the relay nodes can be employed to relay the information for the faraway mobiles so as to maintain the basic QoS. The base station is in charge of optimize the system performance by scheduling the mobiles' direct transmission and relay transmission. 
The optimization problem lies in two factors. First, we should decide how many relay nodes are necessary to help each user. We denote $N_{i}$ as the number of relay nodes for user $i$. Second, we should decide who should relay the information. We should also determine the modulation scheme $M_{i}$ used by user $i$. Hence the resources allocated to each user are the number of relays to help him and the modulation scheme. Suppose we fix the BER for all users in the system. From (17), there is a tradeoff between each user's rate and the number of relays. If there is more number of relays, with the same BER, the user can transmit a higher rate. However the user needs more time slots for the relay transmission. So the user $i$ 's rate can be written as:

$$
T_{i}=\frac{\log _{2} M_{i}}{N_{i}+1} .
$$

In order for each user to be able to calculate the rate in (19), we need to obtain the channel information such as $\sigma_{s, d}^{2}$, $\sigma_{r_{i}, d}^{2}$, and $\sigma_{s, r_{l}}^{2}$ in (17). The source to destination channel condition $\sigma_{s, d}^{2}$ can be obtained from the feedback channel from the base station. To obtain the source to relay and relay to the base station channel conditions, one simple method is to broadcast all information. However this method will cause too many communication overheads. To reduce the signaling, we propose the following distributed communication protocol. The basic idea for the distributed communication protocol is to find the route back to the base station. Here we assume that the communication channels are reciprocal. So by listening to the uplink of the relay transmission, the source can know who are the neighbors and what are the channels between the source and relays. By flooding route discovery requests to the neighbor, the source tries to find the optimal route. If the neighbor has a route back to the base station, it will reply to the source with the information such as who are on the route and what are the channels from the relays to the base station. If the neighbor does not have a route, it will flood the request to its neighbor. The maximal number of flooding is limited to reduce the signalling. Moreover, the loop should be prevented from the discovered routes. By using this protocol, the distributed users can obtain the necessary information to optimize (17) and (19).

Next, we discuss the network optimization performed at each individual node and at the base station. Each user obtains the information for his neighbor nodes via the route discovery algorithm described above. Each user then tries to maximize his own throughput given in (19) subject to the BER constraint and a constraint on the maximum number of relays that can help him as follows

$$
\begin{gathered}
\qquad \max _{\mathbf{A}_{i}, N_{i}, M_{i}} T_{i} \\
\text { s.t. } \begin{cases}\text { Error Rate: } & \mathrm{BER}_{i} \leq \delta, \forall i, \\
\text { Maximal Relay: } & N_{i} \leq N_{\gamma}, \forall i, \\
\text { Modulation: } & M_{i} \in \mathcal{M}, \forall i,\end{cases}
\end{gathered}
$$

where $A_{i}$ is a vector of the nearest neighbors to user $i, \delta$ is the maximal BER that system can accept, $N_{\gamma}$ is the maximum number of relays that can be assigned to help any user, and $\mathcal{M}$ is the set of allowed modulation schemes. The problem in
(20) is an integer programming problem and can be shown to be $N P$ hard. All users feedback their optimized parameters to the base station, and the users that can not meet the system requirement are considered in outage. This is a distributed algorithm since the processing is done locally at the users.

\section{Simulation Results}

In this section, we provide some computer simulations to demonstrate the gains achieved by the proposed joint resourceallocation and cooperation protocol. Two measures are used to test the overall system performance, the outage probability and the average rate achieved per user. Two systems will be compared to each other:(i) The non-cooperative system, in which each user forwards his data directly to the basesation without any relaying (single hop), and the (ii) the cooperative scheme in which cooperation between users is employed to forward each others data. For fair comparison between the two systems, the total transmitted power for each case per user's data is kept constant, i.e., if $P_{\max }=P$ is used per user for the non-cooperative case then $P_{\max }=P /(N+1)$ is used per transmitting node for the cooperative scheme if $N$ relays are assigned to help this specific source.

The outage event of the system is defined as the proportion of users that does not satisfy the system constraint. For the non-cooperative case, the only system constraint is the quality of service, which is the user's BER in this case not exceeding a pre-specified threshold which is set to $10^{-2}$ in our simulations. For the cooperative scheme, two constraints must be satisfied: (1) The user's BER not exceeding a pre-spcified theshold $\delta=$ $10^{-2}$; (2) The maximum number of relays allowed to help a user can not exceed a certain threshold that is set to $N_{\gamma}=3$ in our simulations. The system performance metrics, outage probability and average rate, are measured against two system parameters that are the maximum allowable transmitted power $P_{\max }$ per user and the cell size. The number of users in all the simulations are fixed to 100 users. The path loss factor is set to 3 . The locations of the users are generated according to a uniform distribution over the cell.

Fig. 2 depicts the system outage probability versus the maximum power $P_{\max }$. The maximum power is in 100's of $\mathrm{mW}$, and the cell radius is fixed to $6 \mathrm{~km}$. It is clear from the figure that the outage for the no-relay (single hop) case is around 0.5 due to the large cell area. However, the outage for the cooperative scheme is less than 0.1 for most of the power range. Fig. 3 depicts the average rate per user versus the power. The rate of the system is increased by about $5 \%$ from the no-relay case. Figures 4 and 5 depict the system outage and rate versus the cell radius in meters, respectively, for a fixed maximum power of $100 \mathrm{~mW}$. It is clear from both figures that for a fixed outage probability at 0.1 the cell size is increased from about $3.2 \mathrm{~km}$ to $5.9 \mathrm{~km}$ which is about $180 \%$ increase and rate is increased by about $5 \%$ for cell radius above $6 \mathrm{~km}$. For small cell sizes the rate per user is higher for the non-cooperative scheme, however, if we consider the total achieved rate in the network, i.e., taking into account the number of users not in outage, then the performance of cooperative transmission will be superior to that of noncooperative transmission. 


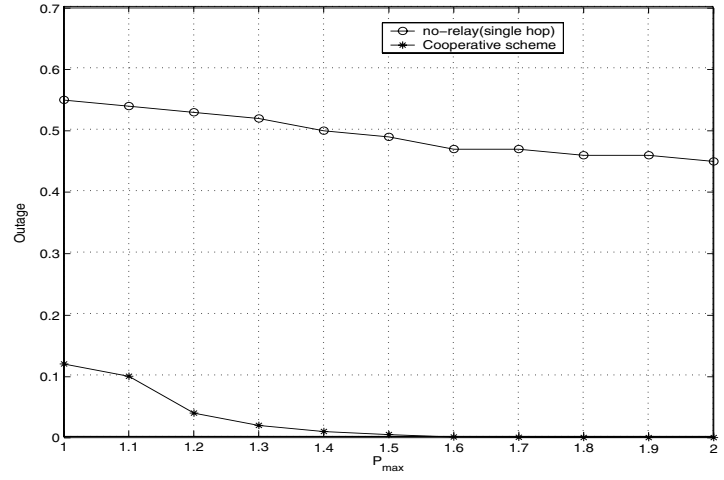

Fig. 2: Outage probability versus $100 \times P_{\max }$ in $\mathrm{mW}$.

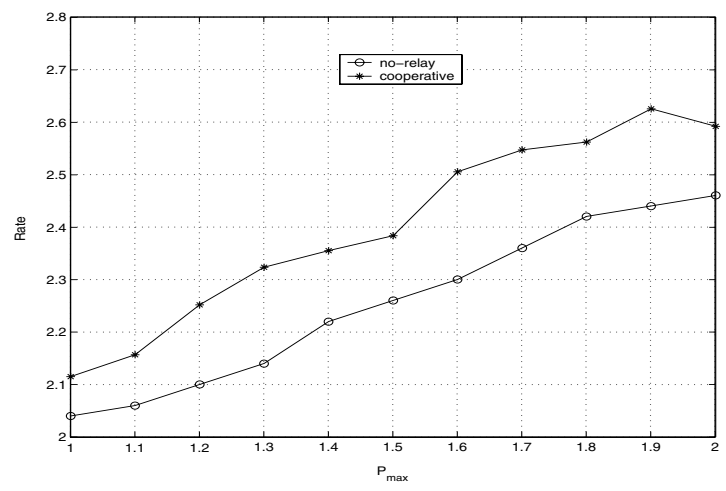

Fig. 3: Rate versus $100 \times P_{\max }$ in $\mathrm{mW}$.

\section{CONCLUSIONS}

In this paper, we presented a resource-allocation strategy for a multi-node cooperative communications protocol. Users can form virtual antenna arrays to send their data to the basestation, hence achieving spatial diversity. Each user tries to maximize his own transmission rate under the constraint of having a maximum number of relays that can help him (hops to the basestation). The total transmitted power is also kept fixed for fairness in comparison with the non-cooperative (single hop) scenario. Exact symbol error rate formulas for the multi-node cooperation protocol are used in the optimization algorithm. The simulation results show that the optimal resource-allocation for the cooperative scheme reduces the system outage considerably compared to the non-cooperative case. Also, our scheme increases the cell-size by about $180 \%$ and allows far away users to be connected to the basestation. Furthermore, our scheme achieves a higher average throughput per user by about $5 \%$ compared to the non-cooperative scheme for large cell sizes.

\section{REFERENCES}

[1] G. J. Foschini and Z. Miljanic. "A simple distributed autonomous power control algorithm and its convergence", IEEE Trans. Vehicular Technology, vol.42, no.4, pp.641646, Nov. 1993.

[2] E. Armanious, D. D. Falconer, and H. Yanikomeroglu, "Adaptive modulation, adaptive coding, and power control for fixed cellular broadband wireless systems", IEEE Wireless Communications and Networking Conference (WCNC'03), 16-20 March 2003, New Orleans, Louisiana, USA.

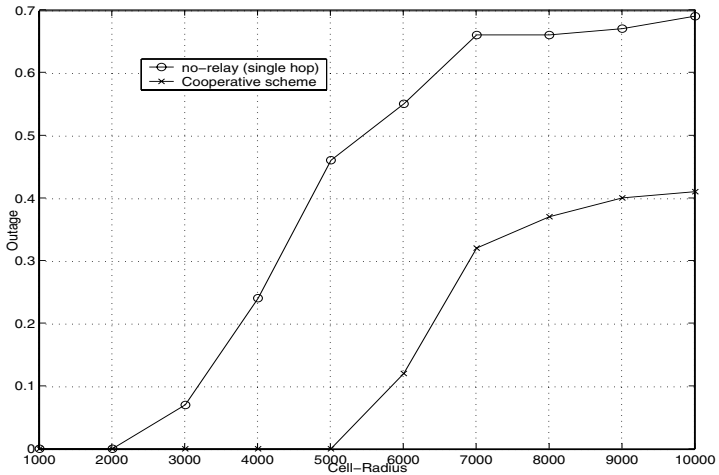

Fig. 4: Outage probability versus cell size measured in cell radius (in meters).

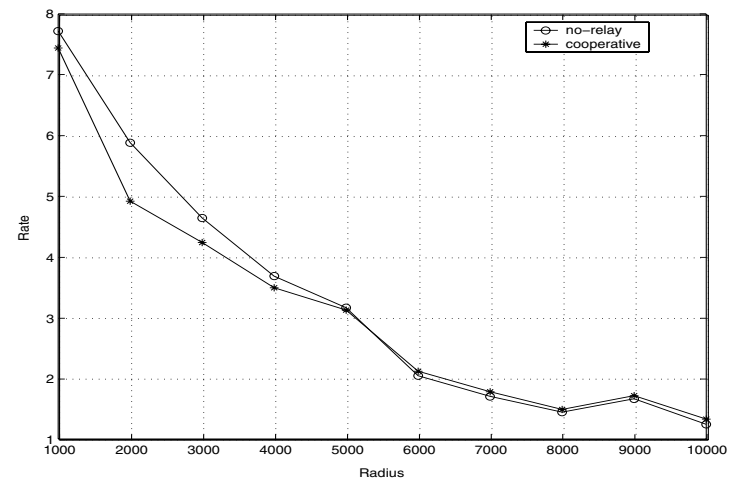

Fig. 5: Rate versus cell size measured in cell radius (in meters).

[3] L. Hanzo, C. H. Wong, and M. S. Yee, "Adaptive wireless transceivers: Turbo-coded, Turbo-equalized and Space-Time coded TDMA, CDMA, and OFDM systems", John Wiley and Sons, 2002.

[4] Z. Han and K.J. Ray Liu, "Power Minimization under Throughput Management over Wireless Networks with Antenna Diversity", IEEE Transactions on Wireless Communications, vol.3, no.6, pp.2170-2181, Nov. 2004.

[5] A. Sendonaris, E. Erkip, and B. Aazhang, "User cooperation diversity, Part I: system description," IEEE Trans. on Commun., vol. 51, no. 11, pp. 1927-1938, Nov. 2003.

[6] A. Sendonaris, E. Erkip, and B. Aazhang, "User cooperation diversity, Part II: implementation aspects and performance analysis," IEEE Trans. on Commun., vol. 51, no. 11, pp. 1939-1948, Nov. 2003.

[7] J. N. Laneman, D. N. C. Tse, and G. W. Wornell, "Cooperative diversity in wireless networks: efficient protocols and outage behavior," IEEE Trans. Inform. Theory, vol. 50, pp.3062-3080, Dec. 2004.

[8] J. N. Laneman and G. W. Wornell, "Distributed space-time coded protocols for exploiting cooperative diversity in wireless networks," IEEE Trans. Inform. Theory, vol. 49, pp.2415-2525, Oct. 2003.

[9] J. Boyer, D. D. Falconer, and H. Yanikomeroglu, "Multihop diversity in wireless relaying channels", IEEE Transactions on Communications, vol. 52, no. 10, pp. 1820-1830, October 2004.

[10] A. K. Sadek, W. Su, and K. J. R. Liu, "A class of cooperative communication protocols for multi-node wireless networks," Proc. IEEE International Workshop on Signal Processing Advances in Wireless Communications (SPAWC), Newyork, June 2005.

[11] D. G. Brennan, "Linear diveristy combining techniques," Proceedings of the IEEE, vol. 91, no. 2, pp. 331-356, Feb. 2003.

[12] M. K. Simon and M. -S. Alouini, "A unified approach to the performance analysis of digital communication over generalized fading channels," Proc. IEEE, vol. 86, no. 9, pp.1860-1877, Sept. 1998 